\title{
Programa Pupunha: software para avaliação econômica da irrigação da pupunha
}

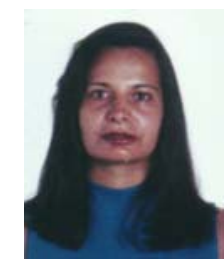

Patricia A. A. Marques ${ }^{1} \&$ Tadeu A. Marques ${ }^{2}$

\author{
1 Av Luciano Guidotti 1260, Bloco 4, Apto. 423, CEP 13424-540, Higienópolis, Piracicaba, São Paulo. Fone: (19) 3426-6509. \\ E-mail: paamarqu@carpa.ciagri.usp.br (Foto)
}

2 Universidade do Oeste Paulista (UNOESTE). E-mail: tmarques@prudenet.com.br

Protocolo $145-24 / 10 / 2001$

\begin{abstract}
Resumo: O programa Pupunha permite a variação dos fatores que interferem no custo da irrigação, favorecendo as condições reais do produtor, através de uma análise direcionada e real da viabilidade de se irrigar a cultura da pupunha (Bactris gasipaes H.B.K). Para se constatar se os custos adicionais da irrigação podem ser pagos pela cultura da pupunha irrigada, o programa calcula o acréscimo de produção necessário para pagar este custo adicional, relacionado à produção de palmito de primeira. São permitidos vários estudos, variando os principais fatores que interferem no custo total anual e, como resultado principal, obtém-se uma família de curvas comparativas para diversas situações, mostrando o acréscimo necessário para vários níveis de produção de palmito de primeira $\left(\mathrm{kg} \mathrm{ha}^{-1}\right)$. Este programa foi realizado para ajudar na tomada de decisão em cultura de pupunha irrigada e está disponível na internet (www.winsite.com/bin/ Info?5000000038400).
\end{abstract}

Palavras-chave: Bactris gasipaes H.B.K, viabilidade econômica, programa

\section{Program Pupunha: Software for economical evaluation of the irrigated pejibaye}

\begin{abstract}
The Pupunha program allows the combinations of the various real conditions of the producer and providing an analysis of the viability of irrigating the pejibaye (Bactris gasipaes H.B.K) crop. In order to evaluate the economics of the additional cost of irrigation; the program calculates the necessary production increment to pay for this additional cost. Several types of studies are possible by varying the principal factors that interfere in the total annual cost. The main result is a family of comparative curves for several situations, showing the necessary production increment for different levels of production of pejibaye $\left(\mathrm{kg} \mathrm{ha}^{-1}\right)$. This program was developed to facilitate decision making with irrigated pejibaye crop and it is available for free at: www.winsite.com/bin/Info?5000000038400.
\end{abstract}

Key words: Bactris gasipaes H.B.K, economical viability, program

\section{INTRODUÇÃO}

Nas regiões onde a insuficiência ou má distribuição das chuvas inviabiliza, em alguns períodos do ano, a exploração agrícola econômica, a irrigação se justifica como recurso tecnológico indispensável ao aumento da produtividade das culturas; entretanto, a viabilidade econômica é um pré-requisito indispensável para sua adoção pelos agricultores (Frizzone et al., 1994). A agricultura irrigada exige alto investimento em obras e aquisição de equipamentos, transporte, controle e distribuição de água, além de gastos com energia e mão-de-obra para operação do sistema, que representam importantes custos adicionais da produção (Azevedo, 1983).

Muitos autores (Ferreira, 1987; Agrianual, 1996; Moro, 1996; Ramos, 1998) relatam que a pupunheira é uma planta exigente em água. Contudo, até o momento existem poucos experimentos sobre a irrigação da pupunha, Clement (1995), nos quais se tenha utilizado irrigação por sulcos, e Defrank \& Clement (1995) que utilizaram aspersores, citam obter bons resultados. Em outros trabalhos, o uso da irrigação promoveu maiores crescimentos (Ramos, 1998) e maiores produtividades (Flori \& D’Oliveira, 1995) porém nenhum desses experimentos era planejado para avaliar a irrigação.

A pupunha tem ampla distribuição geográfica e, em alguns locais, sem o uso da irrigação pode ter sua rentabilidade comprometida (Ferreira, 1987). Segundo Moro (1996) para regiões com mais de dois meses seguidos de déficit hídrico, a irrigação se torna necessária. Furia (1997) cita que, dentre os fatores limitantes à produção do palmito de pupunha, este é um fator contornável, uma vez que se pode dispor de um sistema de irrigação, mas lembra que a instalação de um sistema de irrigação acarreta mais custos, os quais devem ser pagos pelo incremento de produção proporcionado pela irrigação. 
Segundo Bovi (1993) o Brasil detém 95\% do mercado de exportação mundial de palmito, com receitas médias anuais ao redor de 30 milhões de dólares, com tendência a expansão e, ainda, com um mercado interno movimentando ao redor de 180 milhões de dólares. A produção da pupunha pode ser vista também com vantagem social, pois segundo Moro (1996) ela pode ser produzida por pequenos produtores os quais, por sua vez, podem levar uma vida mais digna no campo.

Neste contexto, pretende-se utilizar, no presente trabalho, das mais modernas técnicas da informática, gerando-se um programa computacional, o qual permite a variação dos fatores que interferem no custo da irrigação permitindo, então, estudarse a viabilidade econômica do uso da irrigação na cultura da pupunha (Bactris gasipaes H.B.K).

\section{DESENVOLVIMENTO TEÓRICO}

O programa foi desenvolvido em linguagem de programação Delphi 3 para sistema operacional Microsoft Windows 95. Para se obter o acréscimo de produção necessário para pagar a irrigação, são necessários cálculos de vários fatores econômicos, descritos na seqüência abaixo:

\section{Custos Fixos}

Os custos fixos são aqueles que ocorrem independentemente do número de horas anuais de operação do sistema de irrigação e, para calculá-los $\left(\mathrm{R} \$\right.$ ano $\left.^{-1} \mathrm{ha}^{-1}\right)$ utilizou-se:

a) Fator de Recuperação de Capital (FRC), utilizando-se os juros anuais fornece, um coeficiente que permite, a partir do valor do investimento, calcular o custo fixo anual referente a este investimento (Francisco, 1991; Bernardo, 1995 e Camargo, 1998), este cálculo leva em conta a vida útil do equipamento (anos) e a taxa de juros ao ano (\%) (Eq. 1).

$$
\mathrm{FRC}=\frac{\mathrm{J}(\mathrm{J}+1)^{\mathrm{V}}}{(\mathrm{J}+1)^{\mathrm{V}}-1}
$$

em que:

FRC - fator de recuperação de capital (decimal)

$\mathrm{J}$ - taxa anual de juros (decimal)

$\mathrm{V}$ - vida útil do bem estudado (anos)

b) Custo da Rede Elétrica (CE), que leva em consideração a distância a ser instalada de rede elétrica, o preço da rede elétrica, a área do módulo considerado e a vida útil do equipamento (Eq. 2).

$$
\mathrm{CE}=\frac{\mathrm{D} P \mathrm{PR}}{\mathrm{A} \mathrm{V}}
$$

em que:

$\mathrm{CE}$ - custo da rede elétrica $\left(\mathrm{R} \$ \mathrm{ano}^{-1} \mathrm{ha}^{-1}\right)$

D - distância da rede elétrica a ser instalada $(\mathrm{km})$

$\mathrm{PR}$ - preço da rede elétrica $\left(\mathrm{R} \$ \mathrm{~km}^{-1}\right)$

A - módulo de área (ha) c) Cálculo do custo fixo anual, que leva em conta o FRC, o CE e o preço de aquisição do sistema (Eq. 3).

$$
\mathrm{CF}=\mathrm{FRC}_{1} \cdot \mathrm{PS}+\mathrm{FRC}_{\text {rede }} \mathrm{CE}
$$

sendo:

$$
\begin{aligned}
& \mathrm{CF} \text { - custo fixo anual }\left(\mathrm{R} \$ \text { ano }^{-1} \mathrm{ha}^{-1}\right) \\
& \mathrm{PS}-\text { preço de aquisição do sistema de irrigação }\left(\mathrm{R} \$ \text { ha }^{-1}\right) \\
& \mathrm{FRC}_{1} \quad \begin{array}{c}
\text { FRC aplicado à vida útil do equipamento de } \\
\text { irrigação (decimal) }
\end{array} \\
& \begin{array}{cc}
\mathrm{FRC}_{\text {rede }} & \begin{array}{c}
\text { FRC aplicado à vida útil da rede elétrica } \\
\text { (decimal) }
\end{array}
\end{array}
\end{aligned}
$$

\section{Custos Variáveis}

No cálculo dos custos variáveis anuais da irrigação, estão envolvidos os custos de energia, de manutenção, de mão-deobra e custo da água (Bernardo, 1995 e Marouelli \& Silva, 1998). Segundo Frizzone (1999) o tipo de motor, elétrico ou a combustão é um parâmetro de grande efeito sobre os custos. Para o motor elétrico é importante considerar-se a modalidade de tarifação elétrica e os custos com a linha de tensão, de acordo com sua distância. Conforme Bernardo (1995) o custo de energia depende também da potência absorvida do motor, número de horas trabalhadas e do consumo médio por hora. Neste trabalho consideraram-se, para cálculo do custo de energia, os motores elétricos e diesel, de acordo com os seguintes cálculos:

a1) No motor elétrico, o Custo de Energia leva em conta a demanda (Frizzone, 2002) e o consumo anual corrigidos pelo ICMS cobrado no Estado (\%) (Eqs. 4, 5 e 6). Em projetos com tensão inferior a $69 \mathrm{kV}$ e demanda de potência igual ou superior a $50 \mathrm{~kW}$ deve-se utilizar a tarifa verde, já projetos com tensão igual ou superior a $69 \mathrm{kV}$ deve-se utilizar a tarifa azul (Frizzone, 2002).

$$
\mathrm{Da}=[(12-\mathrm{M}) 0,1+\mathrm{M}] \mathrm{Td} \mathrm{P} 0,73259(1+\mathrm{ICMS})
$$

em que:

$\mathrm{Da}$ - custo da demanda de energia elétrica anual $\left(\mathrm{R} \$ \mathrm{ano}^{-1}\right.$ $\mathrm{ha}^{-1}$ )

M - meses de operação do sistema de irrigação

$\mathrm{Td}$ - tarifa de demanda $\left(\mathrm{R} \$ \mathrm{KW}^{-1}\right)$

$\mathrm{P}$ - potência do motor $\left(\mathrm{cv} \mathrm{ha}{ }^{-1}\right)$

ICMS - imposto sobre a circulação de mercadorias e serviços cobrado na região (decimal)

$$
\mathrm{Ca}=\mathrm{M}[\mathrm{Hd} .(1 \text { - De })+\mathrm{Hs}] \mathrm{Tc} \text { Do P 0,73259 (1 + ICMS })
$$

em que:

$\mathrm{Ca}$ - custo de consumo de energia elétrica anual $\left(\mathrm{R} \$\right.$ ano $^{-1}$ ha $^{-1}$ )

Hd - horas com desconto (23 às $5 \mathrm{~h}$ ) (Frizzone, 1999)

De - desconto da Portaria 105 DNAEE (decimal)

Hs - horas sem desconto

$\mathrm{Tc}$ - tarifa de consumo $\left(\mathrm{R} \$ \mathrm{kWh}^{-1}\right)$

Do - dias de operação por mês 
O custo anual da energia (Eq. 6) pode ser expresso pela soma dos faturamentos da demanda e de consumo, ajustados pelo fator de potência (Frizzone, 1999)

$$
\mathrm{Cen}=(\mathrm{Da}+\mathrm{Ca})\left(\frac{0,92}{\cos \varphi}\right)
$$

em que:

Cen - custo de energia $\left(\mathrm{R} \$ \mathrm{ano}^{-1} \mathrm{ha}^{-1}\right)$

$\cos \varphi$ - fator de potência, referente a defasagem entre corrente e tensão na instalação elétrica (Frizzone, 2002)

a2) No motor diesel, o Custo de Energia considera os meses de operação por ano, o número de dias irrigados por mês, o número de horas operando por mês, a potência do motor, o consumo específico do motor e o preço do óleo diesel na propriedade (Eq. 7).

$$
\text { Cen }=\text { Co P Cs 0,00125 H Do M }
$$

em que:

Co - custo do óleo diesel na propriedade $\left(\mathrm{R} \$\right.$ litro $\left.^{-1}\right)$

$\mathrm{Cs}$ - consumo específico (gramas de diesel $\mathrm{CV}^{-1} \mathrm{~h}^{-1}$ )

H - horas de operação por dia

b) O Custo de Manutenção considera uma porcentagem do custo de Energia (Frizzone, 1999) a qual é um valor de entrada no programa (Eq. 8).

$$
\mathrm{Cm}=\frac{\% \quad \text { Cen }}{100}
$$

em que:

$\mathrm{Cm}$ - custo de manutenção ( $\left.\mathrm{R} \$ \mathrm{ano}^{-1} \mathrm{ha}^{-1}\right)$

c) O Custo da Mão-de-Obra considera o salário mensal médio pago, o número de funcionários e os encargos pagos sobre o salário, resultando em $\mathrm{R} \$ \mathrm{ano}^{-1} \mathrm{ha}^{-1}$ (Eq. 9) (Marquelli \& Silva, 1998 e Frizzone, 1999).

$$
\mathrm{MO}=\mathrm{S}\left(1+\left(\frac{\mathrm{F}+\mathrm{T}+\mathrm{I}+\mathrm{IT}}{100}\right)\right) \mathrm{Nf}
$$

onde:

MO- custo da mão-de-obra $\left(\mathrm{R} \$ \mathrm{ano}^{-1} \mathrm{ha}^{-1}\right)$

$\mathrm{S}$ - salário mensal $\left(\mathrm{R} \$ \mathrm{mês}^{-1}\right)$

$\mathrm{F}$ - encargo pago relativo a férias em porcentagem do salário $(\%)$

$\mathrm{T}$ - encargo pago relativo ao $13^{0}$ salário em porcentagem do salário $(\%)$

I - encargo pago relativo ao INSS em porcentagem do salário (\%)

IT - encargo pago relativo ao INSS do $13^{0}$ salário em porcentagem do salário (\%)

$\mathrm{Nf}$ - número de funcionários para o manejo da irrigação (homens ha-1) d) O Custo da Água, quando cobrada, pondera a evapotranspiração da cultura, dias irrigados por mês, número de meses de operação do sistema por ano e o preço da água $\left(\mathrm{R} \$ \mathrm{~m}^{-3}\right)(\mathrm{Eq} .10)$.

$$
\mathrm{Ca}=\mathrm{ETc} \text { Do M Pa } 10
$$

em que:

$\mathrm{Ca}$ - custo da água $\left(\mathrm{R} \$ \mathrm{ano}^{-1} \mathrm{ha}^{-1}\right)$

ETc - evapotranspiração da cultura $\left(\mathrm{mm} \mathrm{dia}^{-1}\right)$

$\mathrm{Pa}$ - preço da água $\left(\mathrm{R} \$ \mathrm{~m}^{-3}\right)$

e) Custo Variável Anual (CV) (Eq. 11).

$$
\mathrm{CV}=\mathrm{Cen}+\mathrm{Cm}+\mathrm{Ca}+\mathrm{MO}
$$

em que:

$\mathrm{CV}$ - custo variável anual $\left(\mathrm{R} \$ \mathrm{ano}^{-1} \mathrm{ha}^{-1}\right)$;

\section{Custo Total}

O Custo Total é a soma dos custos fixos anuais e dos custos variáveis anuais (Eq. 12).

$$
\mathrm{CT}=\mathrm{CF}+\mathrm{CV}
$$

em que:

$$
\text { CT - Custo Total anual ( } \left.\mathrm{R} \$ \mathrm{ano}^{-1} \mathrm{ha}^{-1}\right) \text {. }
$$

\section{Renda}

Segundo Frizzone (1999) a receita bruta, utilizada em uma análise financeira, é obtida pela multiplicação da produtividade agrícola pelo preço pago. Assim a renda anual foi calculada considerando-se a produção de palmito de primeira e o preço do palmito no mercado interno (Eq. 13).

$$
\mathrm{R}=\mathrm{Pp} \text { Ppal }
$$

em que:

$$
\begin{aligned}
& \mathrm{R} \text { - renda anual }\left(\mathrm{R} \$ \mathrm{ano}^{-1} \mathrm{ha}^{-1}\right) \\
& \mathrm{Pp} \text { - produção de palmito de primeira }\left(\mathrm{kg} \mathrm{ha}^{-1} \mathrm{ano}^{-1}\right) \\
& \text { Ppal - preço do palmito de primeira }\left(\mathrm{R} \$ \mathrm{~kg}^{-1}\right)
\end{aligned}
$$

\section{Acréscimo Necessário de Produção}

Calculou-se este acréscimo (Eq. 14) baseado no Custo Total Anual da irrigação obtido e no acréscimo da Renda obtida para pagá-lo. Para cada produção média obteve-se um acréscimo necessário de produção, resultando em um par ordenado, o qual originou um ponto no gráfico e o conjunto de pontos do referido gráfico apresenta uma curva para cada nível do fator estudado (Fig. 6). A lucratividade esperada está relacionada ao incremento dos gastos com adubação, corte e outros, decorridos pelo aumento da produtividade, devido à irrigação.

$$
\text { Acréscimo de Produção }=\left(\frac{\mathrm{CT}}{\mathrm{R} \frac{\mathrm{L}}{100}}\right) 100
$$

em que:

L - Lucratividade esperada (\%) 


\section{DESCRIÇÃO DO PROGRAMA}

Para uso prático obteve-se o Programa Pupunha, que permite as combinações dos recursos do produtor e uma análise mais direcionada e, conseqüentemente, mais real da viabilidade de irrigar ou não a cultura, em suas condições. O programa está disponível na internet no site www.winsite.com/bin/Info?5000000038400, sendo que o mesmo apresenta exemplos prontos, os quais podem ser abertos e estudados para fins de melhor compreensão de sua utilização. Para se constatar se os custos adicionais da irrigação podem ser pagos pela cultura da Pupunha, o programa os calcula e fornece o acréscimo de produção necessário para pagar este custo anual, relacionado à produção de palmito de primeira. Vários tipos de combinação, podem ser estudados, variando os principais fatores que interferem no custo total anual e, como resultado principal, obtém-se uma família de curvas comparativas, mostrando o acréscimo necessário de produção de palmito de primeira.

A janela principal permite abrir-se um arquivo já existente ou criar novo arquivo (Fig. 1) enquanto a próxima janela admite a escolha do fator variável e o número de níveis deste fator variável (Fig. 2).

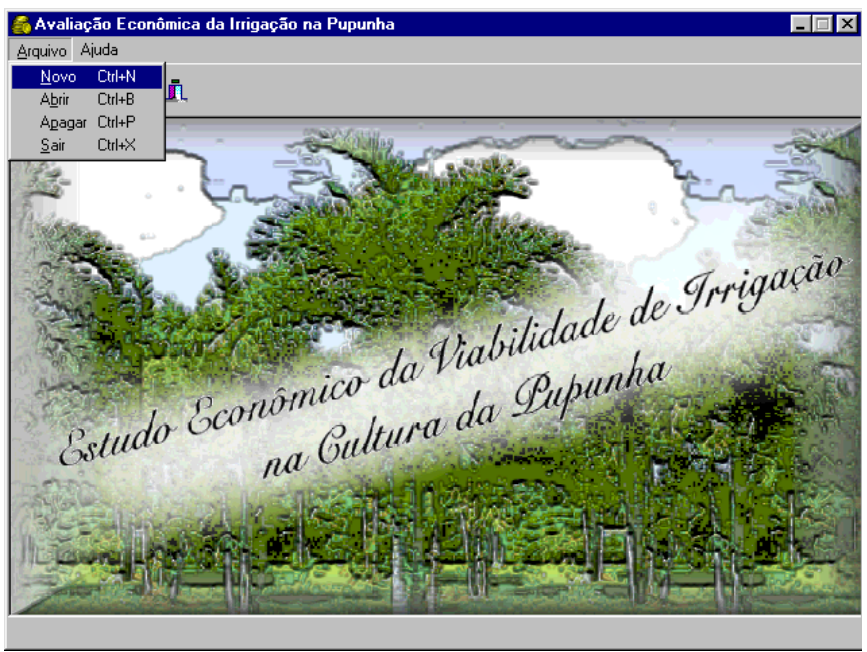

Figura 1. Janela principal do programa Pupunha

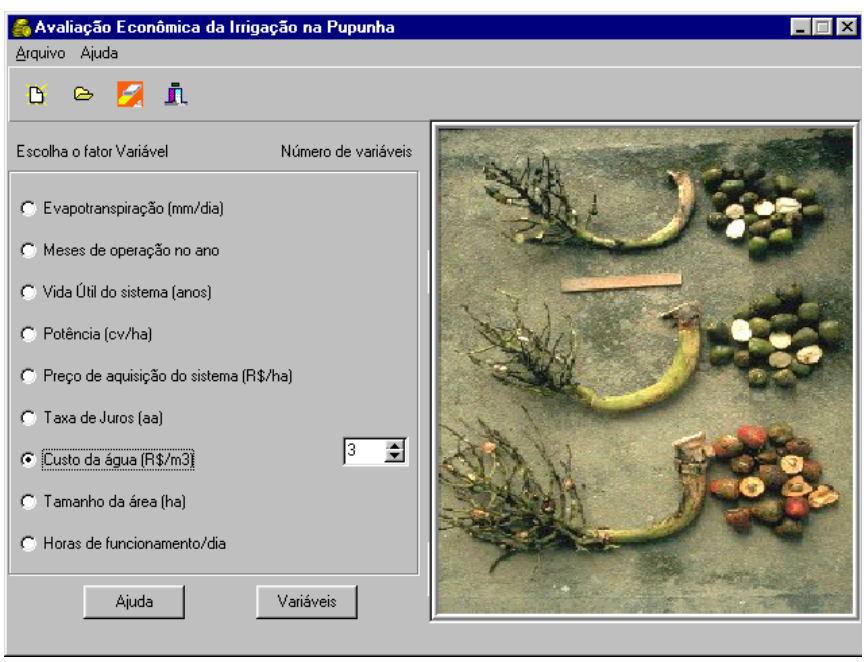

Figura 2. Escolha do fator variável
Após se escolher o fator variável, tem-se a janela para preenchimento dos fatores fixos e dos níveis do fator variável (Fig. 3) e, em seqüência, a janela de preenchimento dos dados fixos (mão-de-obra, lucratividade, produtividade de sequeiro etc.) e escolha do tipo de motor (elétrico ou diesel); esta janela pode ser vista na Figura 4.

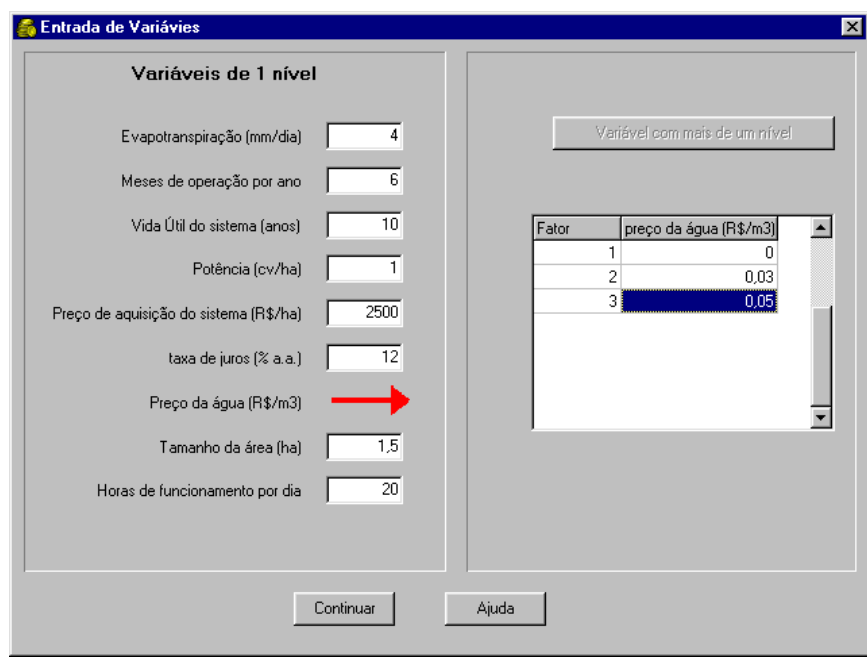

Figura 3. Janela de preenchimento dos fatores fixos e dos níveis do fator variável

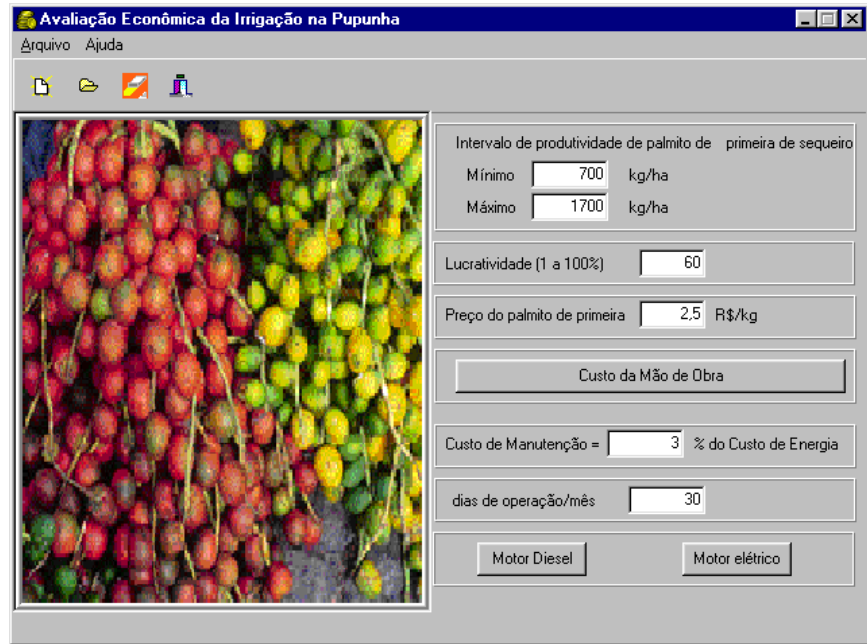

Figura 4. Janela de preenchimento dos dados fixos

Com todos os dados preenchidos, o programa calcula os fatores econômicos referentes e gera o custo Total Anual para cada nível do fator variável e os acréscimos de produção necessários para pagar o custo Total Anual de cada nível para o intervalo de produtividade de sequeiro (Fig. 5); em seguida, é plotado o gráfico referente ao estudo, permitindo sua cópia para a área de transferência do computador (Fig. 6).

Para se resolver dúvidas sobre os cálculos ou sobre alguma variável, existe a opção Ajuda no menu principal, que possui a opção das Fórmulas utilizadas e o Help (Fig. 7).

\section{APLICAÇÃO}

Para o exemplo de aplicação selecionou-se na "janela de escolha" o fator variável como o custo da água, clicando-se no 


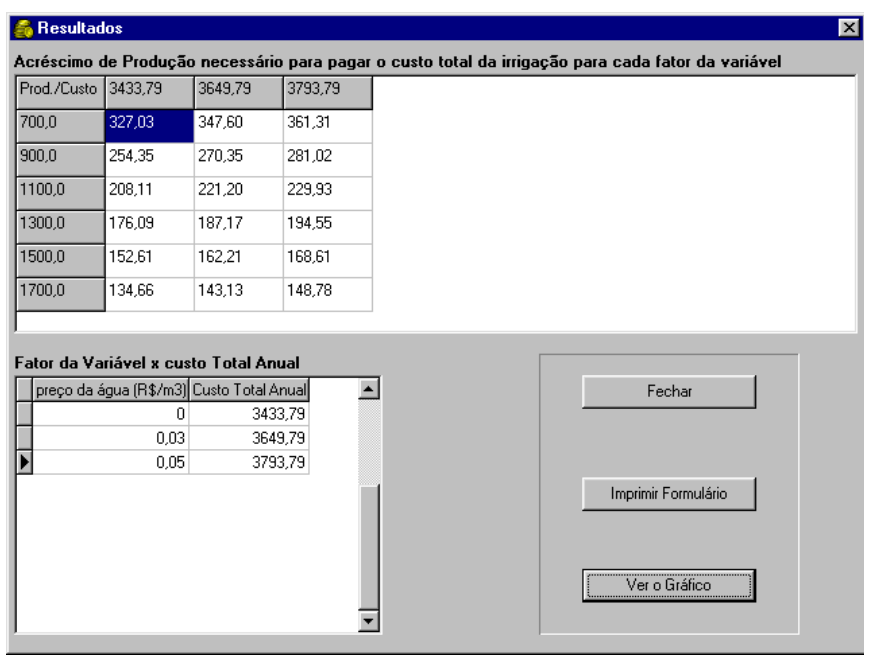

Figura 5. Janela de resultados

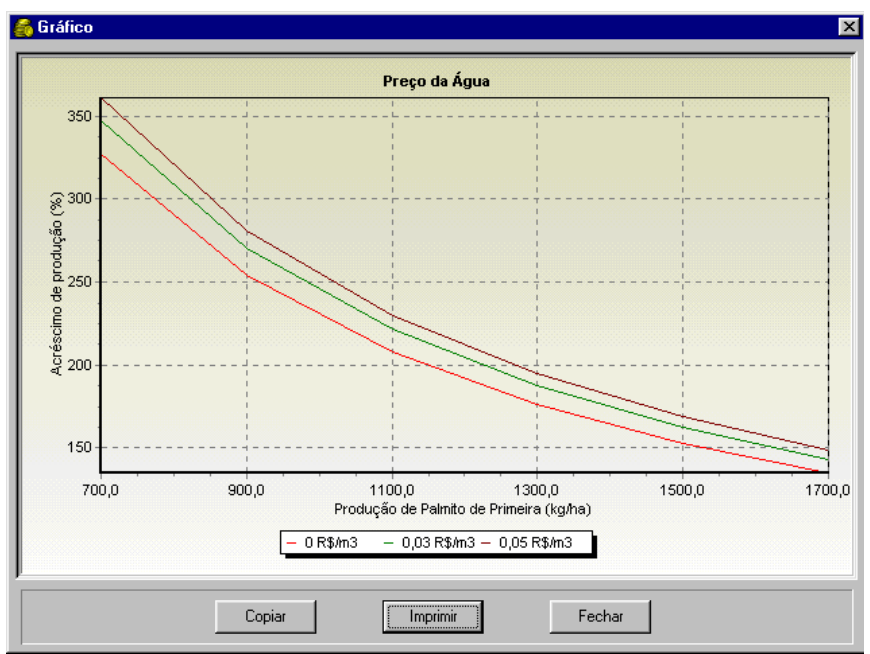

Figura 6. Janela do gráfico de resultados

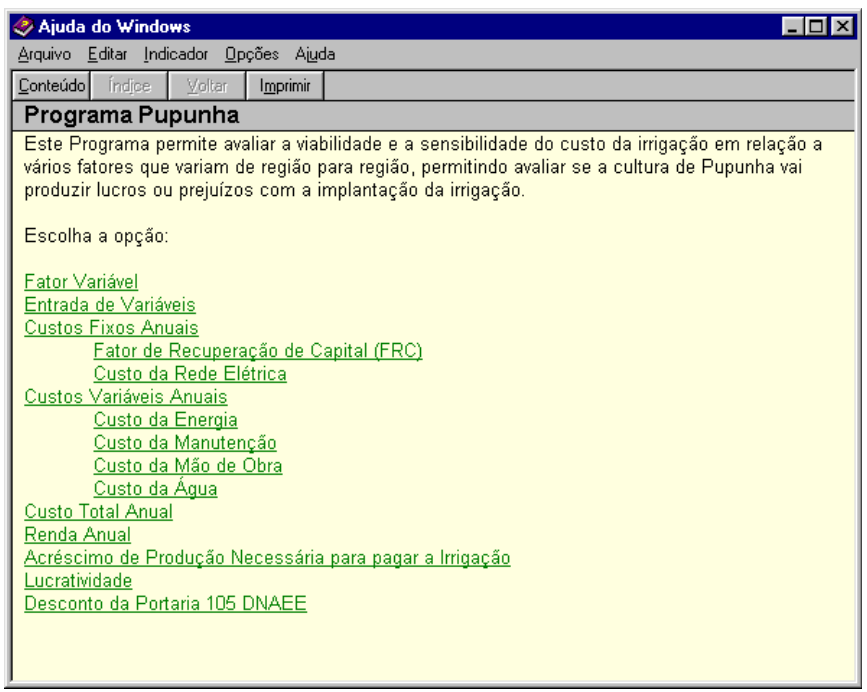

Figura 7. Help do programa Pupunha

botão de seleção situado à frente desta variável; logo após esta operação, surge o campo para definição de quantos níveis terá este fator variável, onde escolheu-se três níveis (Figura 2). Em seqüência escolheu-se o "botão variáveis", para permitir a entrada dos valores referentes ao custo da água, observa-se na Figura 2, que todos os campos permitem apenas uma entrada, com exceção do fator "Custo da água", o qual aparece encoberto por uma seta vermelha, a qual sinaliza que os valores devem ser preenchidos no lado direito da janela, onde aparecem três níveis para esta variável. Em seguida, definiram-se os valores das variáveis fixas (Fig. 3) sendo: evapotranspiração de $4 \mathrm{~mm} \mathrm{~d}^{-1} ; 6$ meses de operação por ano; vida útil de 10 anos; potência de $1 \mathrm{cv} \mathrm{ha}^{-1}$; preço de aquisição do sistema de $\mathrm{R} \$ 2500 \mathrm{ha}^{-1}$; taxa de juros de $12 \%$ ao ano; área de 1,5 ha e $20 \mathrm{~h}$ de funcionamento do sistema por dia. Para o fator variável custo de água, os valores $\mathrm{R} \$ 0,00 \mathrm{~m}^{-3}, \mathrm{R} \$ 0,03 \mathrm{~m}^{-3} \mathrm{e} \mathrm{R} \$ 0,05 \mathrm{~m}^{-3}$ foram utilizados. Posteriormente escolheu-se o "botão continuar", o qual conduziu ao formulário dos demais fatores fixos (Fig. 4), onde foram inseridos: faixa de produtividade do palmito de sequeiro de $700 \mathrm{~kg} \mathrm{ha}^{-1}$ a $1700 \mathrm{~kg} \mathrm{ha}^{-1}$; a lucratividade em $60 \%$; o preço do palmito de primeira $\mathrm{R} \$ 2,50 \mathrm{~kg}^{-1}$; custo de manutenção de $3 \%$ e $30 \mathrm{~d}$ de operação por mês. Em seqüência escolheu-se o botão "custo da mão de obra", onde adicionou-se os valores relacionados a quantidade de funcionários e salários, sendo: 0,5 funcionário ha ${ }^{-1} \mathrm{e}$ salário de 130,00 R $\$$ mês $^{-1}$, respectivamente. Escolheu-se o motor elétrico, pressionando o botão relativo a esta opção. Assim feito surge o botão calcular.

Após realizados os cálculos, podem ser vistos, na Figura 5, os valores de acréscimo necessário de produção para pagar os custos adicionais da irrigação, sendo um valor para cada combinação de Custo Total e produtividade de sequeiro. Nesta Figura, também podem ser vistos os Custos Totais obtidos para cada valor do fator variável, no caso o custo da água. $\mathrm{Na}$ Figura 6, tem-se o gráfico com as curvas comparativas, no qual cada curva se refere a um nível do fator variável.

Neste exemplo, observou-se (Fig. 5) que, em função da produtividade de sequeiro, necessita-se de determinado acréscimo mínimo na produtividade do palmito de primeira, quando do uso da irrigação, para pagar os custos adicionais advindos da mesma. Se o seu uso promover um acréscimo de produtividade maior que os valores mínimos calculados pelo programa, para cada situação estudada, viabilizar-se-á o uso da irrigação.

\section{CONCLUSÕES}

1. Através de dados regionais, é possível se estudar os fatores reais do produtor que interferem no Custo Total Anual da Irrigação.

2. O Programa Pupunha facilita a decisão do uso ou não da irrigação, com base em dados econômicos, representando assim, uma ferramenta de tomada de decisões. Trata-se de um programa de fácil utilização e que permite o salvamento dos dados para análises posteriores.

\section{LITERATURA CITADA}

AGRIANUAL. Palmito: da extração ao cultivo. In: Anuário estatístico da agricultura brasileira. São Paulo: FNP/M\&S, 1996. p.307-312. Agrianual 97 
Azevedo, J.A. Aspectos sobre o manejo da irrigação por aspersão para o cerrado. Brasília: EMBRAPA/CPCA, 1983. 53p. Circular Técnica, 16

Bernardo, S. Manual de irrigação. 6ed. Viçosa: UFV, 1995. 657p.

Bovi, M.L.A. Palmito Pupunha: Informações básicas para o cultivo. In: Camargo, G.M.S.; Furia, L.R.R. (coord.) Encontro sobre a produção de palmito. Piracicaba: CALQ, 1993. p.12-23.

Camargo, I.M.T. Noções básicas de engenharia econômica: aplicações ao setor elétrico. Brasília: FINATEC, 1998. 160p.

Clement, C.R. Growth and genetic analysis of pejibaye (Bactris gasipaes Kunth, Palmae) in Hawaii. Honolulu: University of Hawaii at Manoa, 1995. 221p. PhD Thesis.

Defrank, J.; Clement, C.R. Weed control in pejibaye heart-ofpalm plantations in Hawaii. HortScience, Alexandria, v.30, n.6, p.1215-1216, 1995.

Ferreira, S.A.N. A cultura da pupunheira. Revista Brasileira de Fruticultura, Cruz das Almas, v.9, p.23-28, 1987.

Flori, J.E.; D'Oliveira, L.O.B. O cultivo da Pupunha sob irrigação no semi-árido do Nordeste brasileiro. Petrolina, PE, EMBRAPA-CPATSA, 1995, 3p. Comunicado Técnico, 62

Francisco, W. Matemática financeira. 7ed. São Paulo: Atlas, 1991.319p.
Frizzone, J.A. Planejamento da irrigação uma abordagem às decisões de investimento. Piracicaba: ESALQ/USP, 1999. $110 \mathrm{p}$.

Frizzone, J.A. Análise de decisão de investimento em irrigação. Piracicaba: ESALQ/USP, 2002.394p.

Frizzone, J.A.; Botrel, T.A.; Freitas, H.A.C. Análise comparativa dos custos de irrigação por pivô-central, em culturas de feijão, utilizando energia elétrica e óleo diesel. Engenharia Rural, Piracicaba, v.5, n.1, p.34-53, 1994.

Furia, L.R.R. Pupunha: alternativa para a produção de palmito, cultivo, usos e manejo. Piracicaba: CAMARA/ NASCESALQ, 1997.20p.

Marouelli, W.A.; Silva, W.L.C. Seleção de sistemas de irrigação para hortaliças. Brasília: EMBRAPA, 1998. 16p. Circular Técnica da Embrapa Hortaliças, 11

Moro, J.R. Produção de palmito de Pupunha: Viçosa: Centro de Produções Técnicas, 1996. 28p. Manual no 87

Ramos, A. Desenvolvimento vegetativo da pupunheira (Bactris gasipae Kunth) irrigada por gotejamento em função de diferentes níveis de depleção de água no solo. Piracicaba: ESALQ/USP, 1998. 66p. Dissertação Mestrado 